\title{
Effect of Zero Point Phase Fluctuations on Josephson Tunneling
}

\author{
Gert-Ludwig Ingold ${ }^{1,2}$ and Hermann Grabert ${ }^{2}$ \\ ${ }^{1}$ Institut für Physik, Universität Augsburg, Universitätsstraße 1, D-86135 Augsburg, Germany \\ ${ }^{2}$ CEA, Service de Physique de l'Etat Condensé, Centre d'Etudes de Saclay, F-91191 Gif-sur-Yvette, France \\ ${ }^{3}$ Fakultät für Physik, Albert-Ludwigs-Universität, Hermann-Herder-Straße 3, D-79104 Freiburg, Germany \\ (Received 19 April 1999)
}

\begin{abstract}
In the presence of phase fluctuations the dc Josephson effect is modified and the supercurrent at zero voltage is replaced by a peak at small but finite voltages. It is shown that at zero temperature this peak is determined by two complementary expansions of finite radius of convergence. The leading order expressions are related to results known from the regimes of Coulomb blockade and of macroscopic quantum tunneling. The peak positions and the suppression of the critical current by quantum fluctuations are discussed.
\end{abstract}

PACS numbers: 74.50. $+\mathrm{r}, 05.40 .-\mathrm{a}, 73.23 . \mathrm{Hk}$

The dc Josephson effect allows a Cooper pair current to flow through a superconducting tunnel junction in the absence of an external voltage. The current is determined by the difference $\varphi$ of the condensate phases on the two sides of the junction through $I=I_{c} \sin (\varphi)$ and is limited by the critical current $I_{c}$. While this feature in the currentvoltage characteristic has zero weight, it acquires a finite width due to either thermal or quantum fluctuations of the phase difference. Here, we analyze the role of quantum fluctuations and concentrate on the Josephson peak at zero temperature.

The finite capacitance $C$ of a Josephson junction is a source of fluctuations of the phase difference $\varphi$ and thus of the broadening of the Josephson peak since the charge $Q$ on the capacitance is the conjugate variable to $\varphi$ [1]. At finite voltage $V$, a current can flow only if the tunneling Cooper pairs can loose their excess energy $2 \mathrm{eV}$. This energy can be transferred to the degrees of freedom present in the electromagnetic environment of the junction described by the impedance of the circuit. In order to observe a peak in the current as a function of the voltage, the Josephson junction needs to be voltage biased. This has become possible experimentally only very recently [2]. Since we are interested in the behavior close to the ideal Josephson peak, typical voltages $V$ are much smaller than the gap voltage $\Delta / 2 e$ and quasiparticle excitations can be neglected.

A minimal circuit displaying quantum fluctuations of the phase is shown in Fig. 1 and may be described in terms of the Hamiltonian [3],

$$
\begin{aligned}
H= & \frac{Q^{2}}{2 C}-E_{J} \cos (\varphi) \\
& +\sum_{n=1}^{\infty}\left[\frac{q_{n}^{2}}{2 C_{n}}+\left(\frac{\hbar}{2 e}\right)^{2} \frac{1}{2 L_{n}}\left(\frac{2 e}{\hbar} V t-\varphi-\varphi_{n}\right)^{2}\right] .
\end{aligned}
$$

The first term corresponds to the charging energy and introduces an energy scale $E_{c}=2 e^{2} / C$. The second term describes the tunneling of Cooper pairs through the junction. The Josephson coupling energy $E_{J}$ is related to the critical current by $I_{c}=2 e E_{J} / \hbar$. The third term describes the coupling of the junction to an external impedance modeled by a set of $L C$ circuits and also takes into account an applied voltage $V$.

Summing the perturbative expansion in the Josephson coupling to all orders, one finds the following for the equilibrium Cooper pair current [4]:

$$
\begin{aligned}
I= & 2 e \sum_{n=1}^{\infty}\left(i \frac{E_{J}}{2 \hbar}\right)^{2 n} \sum_{\{\zeta, \eta\}}\left(\prod_{k=1}^{2 n-1} \eta_{k}\right) \zeta_{0} \\
& \times \int_{0}^{\infty} d t_{1} \cdots \int_{0}^{t_{2 n-2}} d t_{2 n-1} \exp (\Gamma),
\end{aligned}
$$

where the exponent in the integrand is given by

$$
\Gamma=i \frac{2 e}{\hbar} V \sum_{k=0}^{2 n-1} \zeta_{k} t_{k}-\sum_{k=1}^{2 n-1} \sum_{l=0}^{k-1} \zeta_{k} \zeta_{l} J\left[\eta_{k}\left(t_{k}-t_{l}\right)\right] .
$$

The sums over $\zeta_{k}$ and $\eta_{k}$ run over the values \pm 1 with the constraint $\sum_{k=0}^{2 n-1} \zeta_{k}=0$.

This result depends on the phase autocorrelation function in the absence of tunneling [3] which at zero temperature is given by

$$
J(t)=2 \int_{0}^{\infty} \frac{d \omega}{\omega} \frac{\operatorname{Re} Z_{t}(\omega)}{R_{Q}}\left(e^{-i \omega t}-1\right) .
$$

It is entirely determined by the total impedance $Z_{t}(\omega)=$ $[i \omega C+1 / Z(\omega)]^{-1}$ seen by the junction. In the following, we assume a purely Ohmic external resistance $R$

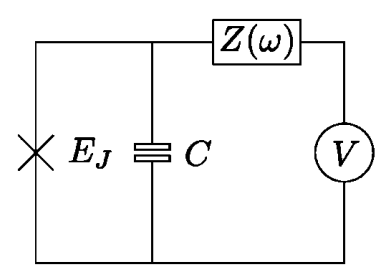

FIG. 1. Josephson junction characterized by the Josephson energy $E_{J}$ and capacitance $C$ coupled to an ideal voltage source $V$ via an external impedance. 
thereby neglecting features in the impedance, such as resonances in a transmission line, which might lead to additional structure in the $I-V$ curve $[5,6]$. One then has

$$
\frac{\operatorname{Re} Z_{t}(\omega)}{R_{Q}}=\frac{\rho}{1+\left(\omega / \omega_{R}\right)^{2}},
$$

where $\rho=R / R_{Q}$ with the resistance quantum $R_{Q}=$ $h / 4 e^{2}$. The total impedance is cut off at a frequency $\omega_{R}=1 / R C$ due to the junction capacitance. For sufficiently long times, the correlation function is given by

$$
J(t)=-2 \rho\left[\ln \left(\omega_{R}|t|\right)+\gamma+i \frac{\pi}{2} \operatorname{sign}(t)\right],
$$

where $\gamma=0.5772 \ldots$ is the Euler constant.

Introducing a dimensionless time $2 \mathrm{eVt} / \hbar$ and making use of the constraint on the $\zeta_{k}$, one finds that for the correlation function (6) each term of the perturbation series (2) depends on the Josephson coupling only through the combination $\left(E_{J} / V^{1-\rho}\right)^{2 n}$. Therefore, the perturbative expansion in $E_{J}$ will finally lead to a power series in the applied voltage $V$. This is a consequence of the special form of the correlation function (4) at zero temperature.

It is instructive to first discuss the problem in several limits where results are already available. We start with the regime of classical phase diffusion which corresponds to taking the limit $\rho=0$. Writing the expansion (2) in terms of a continued fraction [4], one obtains

$$
\frac{I}{I_{c}}=\frac{V}{R I_{c}}-\left[\left(\frac{V}{R I_{c}}\right)^{2}-1\right]^{1 / 2} \Theta\left(\frac{V}{R I_{c}}-1\right),
$$

where $\Theta(x)$ is the Heaviside step function. This corresponds to the zero temperature limit of the result obtained by Ivanchenko and Zil'berman [7]. The current-voltage characteristic (7) starts with an Ohmic line and displays a cusp at $V=R I_{c}$. A further increase of the voltage results in a decreasing current.

As discussed above, an expansion in the Josephson coupling energy at $\rho=0$ amounts to an expansion in $1 / V$. The series (2) corresponds to the Taylor expansion of (7) given by

$$
I=\frac{1}{2 \pi^{1 / 2}} \frac{V}{R} \sum_{n=1}^{\infty} \frac{\Gamma(n-1 / 2)}{\Gamma(n+1)}\left(\frac{V}{R I_{c}}\right)^{-2 n} .
$$

This series has a finite radius of convergence and is restricted to $|V|>R I_{c}$. The limit of convergence just coincides with the position of the cusp. Hence, we see that for small $\rho$ and low temperatures the expansion (2) will converge only for sufficiently large voltages.

To examine this further, we now allow for finite $\rho$ and turn to the regime of charging effects where $E_{c} \gg$ $E_{J}$. Within the standard theory of environmental effects on Coulomb blockade (CB) [3], tunneling is treated perturbatively and the current-voltage characteristics are obtained from the leading term $(n=1)$ of the series (2) [8]. For an environment with an Ohmic low frequency component, one finds a zero bias anomaly of the Cooper pair current [9],

$$
I=\frac{\pi^{1 / 2} \rho}{2 \Gamma(\rho) \Gamma(\rho+1 / 2)} \frac{\left(\pi E_{J}\right)^{2}}{\left(\hbar \omega_{R} e^{\gamma}\right)^{2 \rho}} \frac{V}{R}(e V)^{2 \rho-2} .
$$

While for $\rho>1$ this result describes the suppression of the current by the Coulomb blockade effect, for $\rho<1$ it corresponds to a divergent zero bias conductance. One might hope that higher order terms regularize the divergence, but the discussion of the classical phase diffusion limit suggests that for $\rho<1$ the series is divergent for small $V$. We will show below that this is indeed the case.

Now, for small $\rho$ and small voltages, corrections to the linear part of the current-voltage characteristic (7) arise from macroscopic quantum tunneling (MQT). In this regime the Josephson junction is mostly in its zero voltage state and the voltage drop occurs at the resistor. Occasionally, a phase slip will cause a finite voltage across the junction leading on average to a finite dc contribution. In the overdamped limit $2 \pi^{2} \rho^{2} E_{J} \ll E_{c}$, the current-voltage characteristic is given by [10]

$$
I=\frac{V}{R}\left(1-\frac{\pi}{2 e^{1 / 2}(\pi \rho)^{5 / 2+2 / \rho}} \frac{E_{c}^{2}}{E_{J}^{2 / \rho}}(e V)^{2 / \rho-2}\right) .
$$

Note that the zero bias differential conductance goes to zero for $\rho<1$ where we found a divergence within CB theory. On the other hand, the zero bias differential conductance of (10) diverges for $\rho>1$.

In order to reconcile these findings we make use of the analogy between a Josephson junction and a damped particle in a periodic potential. Schmid [11] has noted that the regions of small and large $\rho$ are related by a self-duality of the model. Further progress $[12,13]$ in the calculation of the mobility of the damped particle has been based on the thermodynamic Bethe ansatz [14]. Duality has also been exploited in the context of the fractional quantum Hall effect $[12,15]$.

It should be emphasized that self-duality relies on strictly Ohmic damping. In contrast, the spectrum (5) has a cutoff frequency $\omega_{R}$. This does not spoil duality in the long-time limit of the correlation function (6) where the dependence on $\omega_{R}$ can be absorbed in an effective voltage scale introduced below. The long-time limit restricts us to small voltages with $e V \ll \hbar \omega_{R}$ or equivalently $V / R I_{c} \ll E_{c} / \pi^{2} \rho^{2} E_{J}$. For the typical case of small environmental impedances, voltages of interest are of order $R I_{c}$, and then a rather wide range of ratios $E_{c} / E_{J}$ is allowed. Below we will show how the $I-V$ curve can be extended beyond the voltage limit imposed by the strictly Ohmic approximation.

As a consequence of duality, the current-voltage characteristics can be obtained from an integral representation $[13,16]$ which implies two complementary expansions describing the zero temperature behavior. In the scaling 
limit the CB series (2) takes the form

$$
I=\frac{V}{R} \sum_{n=1}^{\infty} c_{n}(\rho)\left(\frac{V}{V_{0}}\right)^{2(\rho-1) n} .
$$

On the other hand, the MQT series, with the leading order terms (10), reads

$$
I=\frac{V}{R}\left(1-\sum_{n=1}^{\infty} c_{n}(1 / \rho)\left(\frac{V}{V_{0}}\right)^{2(1 / \rho-1) n}\right) .
$$

The coefficients of these expansions are determined by duality and given by

$$
c_{n}(\rho)=(-1)^{n-1} \frac{\Gamma(1+\rho n) \Gamma(3 / 2)}{\Gamma(1+n) \Gamma(3 / 2+(\rho-1) n)},
$$

and the voltage scale is set by

$$
V_{0}=\frac{\pi E_{J}}{e}\left[\Gamma(\rho)\left(\frac{e^{\gamma}}{\pi^{2} \rho} \frac{E_{c}}{E_{J}}\right)^{\rho}\right]^{1 /(\rho-1)} .
$$

The two expansions (11) and (12) have a finite radius of convergence which can be expressed in terms of a critical voltage

$$
V_{c}=V_{0}\left(|1-\rho| \rho^{\rho /(1-\rho)}\right)^{1 / 2} .
$$

For $\rho<1$ the series (11) converges for $V>V_{c}$ and thus is a large voltage expansion, as discussed above, while the series (12) converges for low voltages $V<V_{c}$. The role of the expansions is interchanged above $\rho=1$ where (11) yields a low voltage expansion while (12) determines the large voltage behavior. The situation is illustrated in Fig. 2 where on the right side it is indicated which expansion converges on which side of the curves.

As we have already seen for the case $\rho=0$, the current-voltage characteristic displays a peak. This is still true for finite $\rho$ as seen from Fig. 3 where we present $I-V$ curves for various values of $\rho$. Here, we show the current as a function of the voltage $V_{J}$ across the Josephson junction which is related to the externally applied voltage by $V_{J}=V-R I$. As can be seen from the figure, for finite external impedance, the $I-V$ curve corresponds to a

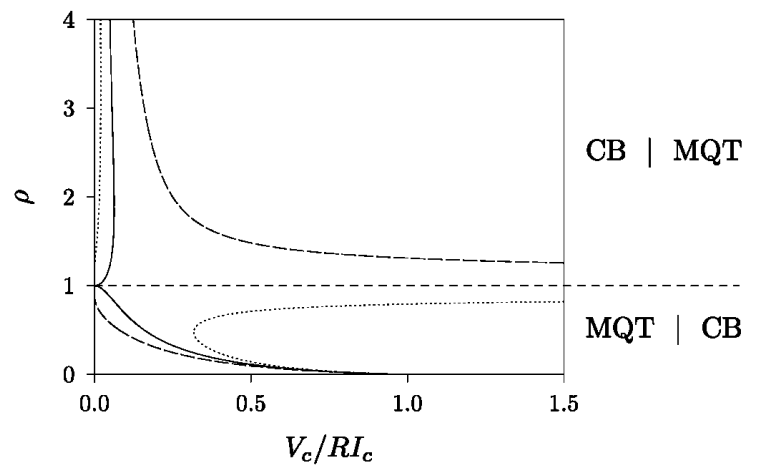

FIG. 2. Range of convergence of the two expansions (11) and (12) for $\left(e^{\gamma} / \pi^{2}\right)\left(E_{c} / E_{J}\right)=0.5,1$, and 2 (dotted, full, and dashed line, respectively). Below the horizontal dashed line $\rho=1$, the series (11) converges to the right of the curves, while above $\rho<1$ it converges to their left. The opposite holds for the series (12). peak of finite width and a maximum current suppressed with respect to the critical current in the absence of fluctuations. As $\rho$ is decreased, the peak narrows and the usual Cooper pair current at zero voltage builds up.

Because of the complicated form of the expansions (11) and (12), an analytic determination of the position and height of the peak in the current-voltage characteristics is, in general, not possible. However, it turns out that to a very good approximation the peak position is given by the critical voltage (15). The quality of this approximation can be seen from Fig. 4 where we compare it (dashed lines) with the peak positions determined numerically (full lines). The lines have been restricted to values of $\rho$ for which $e V_{\max } \leq 10 \hbar \omega_{R}$ in order to ensure the applicability of the theory. Likewise, the maximum current can quite reliably be estimated by $I_{\max } \sim V_{c} / R$. For more precise results, a numerical evaluation of (11) and (12) is required which does not present special problems.

As already emphasized, the range of validity of the expansions (11) and (12) is restricted by the assumption of strictly Ohmic damping. However, the current-voltage characteristics can be extended to larger voltages if $\mathrm{CB}$ theory yields a good description at the limit of validity. One may then continue the $I-V$ curve to larger voltages by using the result of $\mathrm{CB}$ theory with the full frequencydependent impedance (5).

To illustrate this point, we consider the case $\rho=1 / 2$ which for strictly Ohmic damping allows for an exact solution $[17,18]$. Summing up the two expansions (11) and (12), one finds in both cases

$$
\frac{I}{I_{c}}=\frac{\pi E_{J}}{2 E_{c} e^{\gamma}} \arctan \left(\frac{2 E_{c} e^{\gamma}}{\pi E_{J}} \frac{V}{R I_{c}}\right) .
$$

Obviously, this result no longer describes a peak structure as was the case for $\rho<1 / 2$. This is, however, an artifact of the assumption of Ohmic damping. For $\rho=$ $1 / 2$, the plateau value of (16) just corresponds to the value (9) given by CB theory. Beyond the validity of

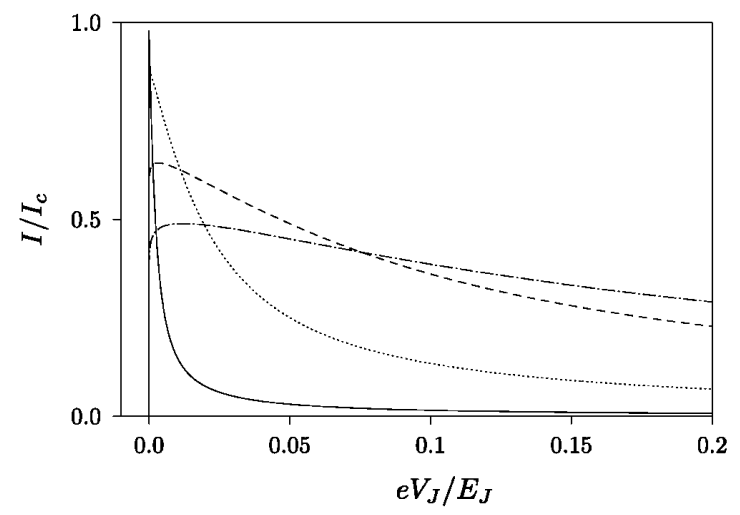

FIG. 3. Zero temperature Cooper pair current-voltage characteristics for $\left(e^{\gamma} / \pi^{2}\right)\left(E_{c} / E_{J}\right)=1$ and $\rho=0.001,0.01,0.05$, and 0.1 shown as full, dotted, dashed, and dash-dotted line, respectively. 


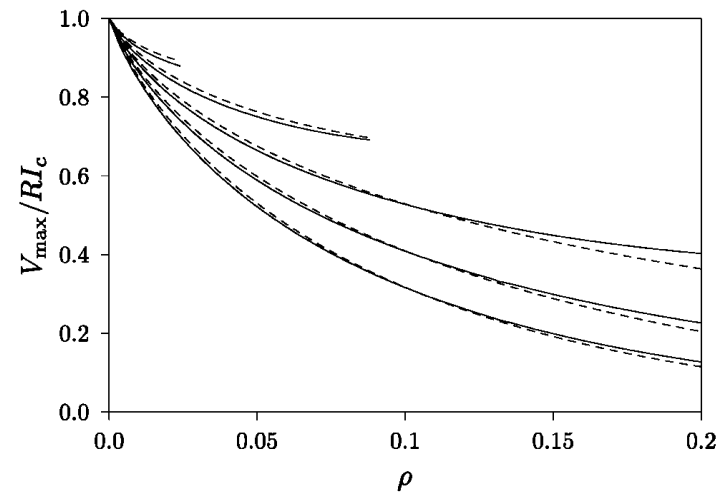

FIG. 4. Comparison of peak positions (full lines) and the critical voltage (15) for $\left(e^{\gamma / \pi^{2}}\right)\left(E_{c} / E_{J}\right)=0.01,0.1,1,10$, and 100 increasing from the upper to the lower curves. Results are shown only for values of $\rho$ which satisfy $e V_{\max } \leq 10 \hbar \omega_{R}$.

the expansions (11) and (12), one may thus use the cutoff-dependent leading order term of the series (2) which describes the decrease of the current for larger voltages and leads again to a peak in the $I-V$ curve. For $E_{c} \gg E_{J}$, (16) reaches its plateau value for very small voltages. Then, $\mathrm{CB}$ theory fails only for voltages below $\left(E_{J} / E_{c}\right) R I_{c}$. Quite generally, for $\rho<1$ and $E_{c} \gg E_{J}$, the result of $\mathrm{CB}$ theory with the exact impedance can be employed except for small voltages. There, however, the strictly Ohmic approximation is appropriate and the results discussed above can be used.

For $\rho>1, \mathrm{CB}$ theory describes the low voltage behavior. For example, for $\rho=2$, the case dual to $\rho=1 / 2$, one finds

$$
\frac{I}{I_{c}}=\frac{V}{R I_{c}}-\left(\frac{E_{c} e^{\gamma}}{4 \pi^{2} E_{J}}\right)^{2} \arctan \left[\left(\frac{4 \pi^{2} E_{J}}{E_{c} e^{\gamma}}\right)^{2} \frac{V}{R I_{c}}\right]
$$

which agrees with (9) to leading order in $V$. This result for strictly Ohmic damping diverges for large $V$ but is in fact regularized by the cutoff in the impedance. Thus, for $\rho>1$ and $E_{c} \gg E_{J}$, the entire peak in the $I-V$ curve is determined by $\mathrm{CB}$ theory.

In conclusion, we have determined the shape of the Josephson current peak in the presence of quantum fluctuations. We have shown that the low voltage behavior can be determined within the approximation of a self-dual model with strictly Ohmic impedance. The results of the dual model have been connected with the phenomena of Coulomb blockade and macroscopic quantum tunneling. This allows for the calculation of the $I-V$ curve for ex- perimentally relevant frequency-dependent impedances in a large range of parameters accessible by state-of-the-art technology.

The authors would like to thank M.H. Devoret, D. Esteve, and A. Steinbach for many inspiring discussions. One of us (G. L. I.) is grateful to the SPEC for hospitality and the Volkswagen-Stiftung for financial support during a stay at the CEA Saclay. The other author (H. G.) was supported by the DAAD through PROCOPE.

[1] M. Tinkham, Introduction to Superconductivity (McGrawHill, New York, 1996).

[2] A. Steinbach, D. Esteve, and M.H. Devoret (private communication).

[3] G.-L. Ingold and Yu. V. Nazarov, in Single Charge Tunneling, edited by H. Grabert and M.H. Devoret, NATO ASI, Ser. B, Vol. 294 (Plenum, New York, 1991).

[4] H. Grabert, G.-L. Ingold, and B. Paul, Europhys. Lett. 44, 360 (1998).

[5] G.-L. Ingold, H. Grabert, and U. Eberhardt, Phys. Rev. B 50, 395 (1994).

[6] T. Holst, D. Esteve, C. Urbina, and M. H. Devoret, Physica (Amsterdam) 203B, 397 (1994); T. Holst, D. Esteve, C. Urbina, and M.H. Devoret, Phys. Rev. Lett. 73, 3455 (1994).

[7] Yu. M. Ivanchenko and L. A. Zil'berman, Zh. Eksp. Teor. Fiz. 55, 2395 (1968) [Sov. Phys. JETP 28, 1272 (1969)].

[8] D. V. Averin, Yu. V. Nazarov, and A. A. Odintsov, Physica (Amsterdam) 165B\&166B, 945 (1990).

[9] M.H. Devoret, D. Esteve, H. Grabert, G.-L. Ingold, H. Pothier, and C. Urbina, Phys. Rev. Lett. 64, 1824 (1990).

[10] S.E. Korshunov, Zh. Eksp. Teor. Fiz. 92, 1828 (1987) [Sov. Phys. JETP 65, 1025 (1987)].

[11] A. Schmid, Phys. Rev. Lett. 51, 1506 (1983).

[12] P. Fendley, A. W. W. Ludwig, and H. Saleur, Phys. Rev. Lett. 75, 2196 (1995); P. Fendley, A. W. W. Ludwig, and H. Saleur, Phys. Rev. B 52, 8934 (1995).

[13] U. Weiss, Solid State Commun. 100, 281 (1996); U. Weiss, in Tunneling and Its Implication, edited by $\mathrm{H}$. Cerdeira, A. Ranfagni, and L.S. Schulman (World Scientific, Singapore, 1997).

[14] Al. B. Zamolodchikov, Nucl. Phys. B 342, 695 (1990).

[15] C. de C. Chamon and E. Fradkin, Phys. Rev. B 56, 2012 (1997).

[16] P. Fendley, Adv. Theor. Math. Phys. 2, 987 (1998).

[17] F. Guinea, Phys. Rev. B 32, 7518 (1985).

[18] U. Weiss and M. Wollensak, Phys. Rev. B 37, 2729 (1988). 